\title{
VRVision: A new tool for the display of 3-D images in behavioral research
}

\author{
JAMES WARD and CHANG HONG LIU \\ University of Hull, Hull, England
}

\begin{abstract}
Researchers using three-dimensional (3-D) scenes in their studies often spend alot of time prerendering the scenes into a set of images for laterpresentations. Toimprove efficiency, we have developed anew plugin program for MATLAB that eliminates this step. The program is ableto manipulate and present 3-D scenes in real time. It allows fine control over numerous parameters, including the mode of projection, angular rotation, lighting, surface photo textures, and animation. Images can be presented with monoscopic or stereoscopic display. It can also dynamically track viewer position and update and display an appropriate 3 -D projection. The program is fully compatible with existing MATLAB utilities such as the Psychophysics Toolbox (psychtoolbox.org). It is made freely available to the research community (www.hive.hull .ac.uk/software/vrvision).
\end{abstract}

Behavioral research often involves presenting 3-D objects or scenes on a computer monitor. Typically, this is achieved by rendering 3-D objects into images and storing the images as a set of stimuli. A great range of 3-D rendering software can be used for this purpose. For example, Liu, Collin, and Chaudhuri (2000) used Geomview (http://www.geomview.org/) to create a bank of face stimuli containing different poses and lighting directions from 3-D face models. Liu and Chaudhuri (2003) used the 3-D rendering functions in MATLAB (MathWorks, Natick, MA) to manipulate the distance between 3-D models and a simulated camera. Others have developed their own programs to create a set of images from 3-D object data. A common feature of these approaches is that they demand considerable time and effort to prepare stimuli for a particular study. In many cases, the created images (often hundreds or even thousands of them) are not easily reusable in future studies.

To improve efficiency of research, we have developed the VRVision software, which is able to display arbitrary 3 -D scenes in real time, with controllable lighting, perspective, and photo texturing. Apart from these basic features, VRVision can also present scenes stereoscopically and can track the viewer's head position to dynamically update and display an appropriate 3-D projection. With VRVision, it is no longer necessary to spend time rendering and creating sets of stimuli prior to experiments.

VRVision is a generic research tool, designed primarily for use by psychologists. There are some cases for which it

This research was supported in part by a grant from the British Academy. The face model used in the examples section was obtained from the University of South Florida (USF HumanID 3-D Face Dataset, http:// marathon.csee.usf.edu/HumanID/). Correspondence concerning this article should be addressed to C. H. Liu, Department of Psychology, University of Hull, Cottingham Road, Hull HU6 7RX, England (e-mail: c.h.liu@hull.ac.uk). is not suitable at the moment, such as certain psychophysical research that requires high precision in illumination, where custom-developed software may still be needed. However, it has the potential to be useful in a wide range of behavioral research studies. The software is accompanied by documentation and examples, so that a user can quickly evaluate whether it is suitable for a particular study.

The program integrates with MATLAB, already a popular and widely used tool in behavioral research, enabling the user to script the course of an experiment from within MATLAB, calling the VRVision module, when it is required, to display stimuli.

VRVision is designed for full-screen use in an experimental setting. It offers new features, such as stereoscopy and head tracking, which are not available in existing plugins, such as the MATLAB Virtual Reality Toolbox 3.1 (MathWorks). VRVision may be combined with the popular Psychophysics Toolbox (Brainard, 1997; Pelli, 1997) for example, to display two-dimensional (2-D) images or to provide on-screen instructions during an experiment.

With the ability of VRVision to dynamically vary conditions, it can provide a way to implement experiments without the need to develop custom software. For example, vision researchers can ask participants to move a target in 3-D to match the depth of a reference point; memory researchers can study the effect of active exploration on object recognition by allowing participants to rotate and manipulate objects with a mouse or a joystick.

In this article, we first will provide a summary of the basic features of the software. We then will describe its additional features, including stereoscopic display and head tracking. Finally, we will describe how to use the software, with some specific examples.

\section{Basic Features}

VRVision displays 3-D scenes loaded from one or more files. The files typically consist of either detailed polygo- 
nal models or compositions of object primitives, such as cubes, cones, spheres, and cylinders. Objects can have associated texture maps, and the texture quality and mapping functions can be specified in the scene file. There is support for transparency and multiple light sources, including spotlights and directional lights.

Scenes can be loaded from Open Inventor (Wernecke, 1994) or VRML files. Other file formats can be converted by using third-party applications - for example, 3D Studio MAX (Discreet/Autodesk Inc.).

The scene position and scale on the screen can be controlled from within MATLAB, from the configuration file of VRVision, or from the scene file itself.

Animation can be embedded within the scene file and will play within the viewer. For example, an object can be made to rotate automatically or to appear and disappear with adjustable timing.

The timing functions of VRVision include the ability to display a scene for a specified time period and to measure the response time between onset of the stimulus and keypresses. The user can specify a particular set of acceptable response keys, and the responses (keypresses) can be detected and recorded.

VRVision is a MATLAB MEX plug-in that provides a number of functions that can be called from within a MATLAB script. It can be started from within a MATLAB program or from the MATLAB console. The program is highly configurable. Many of the settings and parameters that are used by VRVision can be set from within MAT$\mathrm{LAB}$, either interactively at the console or from within a MATLAB script. The settings that are less frequently altered - for example, tracking system settings - are adjusted through a configuration file.

Although VRVision was developed within MATLAB 6.5, it is expected to work with later versions also. It does not depend on any additional MATLAB toolboxes.

At present, VRVision runs only on Microsoft Windows 2000 and XP, although a Mac OS X version is planned in the future.

\section{Stereoscopic Display}

VRVision provides support for stereoscopic 3-D display on appropriate hardware. This makes it possible to display an object outside the screen surface (negative parallax), on the screen surface, or inside the screen (positive parallax). This is achieved by altering the object scale and position, relative to the screen.

To achieve a correct stereoscopic projection, it is generally recommended that an asymmetric viewing frustum (off-axis projection) be used (Akka, 1998). This is the approach that VRVision uses when projecting stereo images. It is also used when head tracking is enabled, to provide an off-axis projection based on the current head position of the viewer.

There is no complex calculation required to achieve a good stereo effect, since the program calculates the appropriate view to display from a few straightforward parameters. These include (1) the monitor width and height,
(2) the viewer position relative to the monitor, which is calculated automatically if the head-tracking mode is enabled, (3) the position and scale of the 3-D scene, and (4) the interpupillary distance, which is required when a stereoscopic display is used.

If care is taken to measure these parameters correctly, VRVision will provide a more accurate stereoscopic projection. The realism can be further improved by enabling the head-tracking facility, since this takes into consideration the current view position of the user and introduces motion parallax cues.

Stereoscopic display hardware is now readily available at a reasonable cost. Typically, this would comprise a suitable graphics card, a CRT monitor, and a pair of LCD shutter glasses.

For stereoscopic support in VRVision, the following hardware is recommended. (1) The display monitor must have a high vertical refresh rate-preferably, at least $100 \mathrm{~Hz}$ - to minimize flicker. Typically, only CRT monitors with short persistence phosphors are suitable. (2) The viewer wears LCD shutter glasses that are synchronized with the display. Examples include the CrystalEyes-3 (StereoGraphics Corp.) or NuVision 60GX (MacNaughton Inc.). (3) Finally, the graphics card must have good OpenGL stereo support and high performance (depending on scene complexity). It must also be compatible with the shutter glasses. Examples are the Quadro-FX (nVidia Corp.) or the Wildcat-4 (3-Dlabs Inc.).

The rendering quality can be greatly improved by selecting a graphics card that is capable of performing full scene antialiasing (FSAA) within OpenGL. This reduces the jagged appearance of polygon edges - typically, by rendering multiple samples for each pixel. This is now a widely supported feature on workstation graphic cards. For example, the Quadro-FX allows the number of antialias samples to be adjusted by the user. Increasing the number of samples will improve quality at the cost of performance.

As an alternative to CRT displays, there are now a number of flat panel auto-stereoscopic displays available on the market. These have the advantage of not requiring glasses. Manufacturers include StereoGraphics Corp., SeeReal Technologies, Dimension Technologies, 4D-Vision, and Sharp Electronics.

Many flat panel auto-stereoscopic displays are likely to be compatible with VRVision (specifically, those that support OpenGL quad-buffer stereo), although this has yet to be confirmed by testing. However, certain flat panel displays exhibit problems, such as a limited range of viewing angles, that might make them unsuitable for experimental work at present.

\section{Head Tracking}

When a 3-D scene is presented on a 2-D display surface such as a monitor, a particular projection must be chosen. This depends on the position of the viewer relative to the monitor. In most cases, this viewing position is either fixed at a predefined point or controlled with a mouse, trackball, or other input device. 
Even in cases in which the viewing position can be altered, the projection itself is usually fixed. Effectively, the object is moved or rotated under a fixed camera. However, if the head position of the viewer is tracked - for example, with an ultrasonic, optical, or electromagnetic tracking system-it is possible to dynamically recalculate and display an appropriate projection based on the current viewpoint.

VRVision directly supports head tracking, provided that an appropriate tracking system is available. To use this feature, a small tracking target is normally attached to the LCD shutter glasses, and the tracking system is configured so that the viewer position can be measured relative to the display monitor. VRVision uses this data to calculate and display the appropriate projection in real time.

In this way, it is possible for the user to view the scene from different viewpoints, simply by using natural head movement. This is particularly effective when combined with the stereoscopic display mode.

At this time, VRVision supports nine different tracking systems, including electromagnetic systems such as the Fastrak (Polhemus Inc.) and Flock of Birds (Ascension Technology Corp.), the ultrasonic 3-DMouse (Logitech Inc.), and several optical tracking systems, including very large volume trackers, such as the Vicon Optical Motion Capture System.

An example of a typical experimental setup that combines stereoscopic display with head tracking is shown in Figure 1. Magnetic tracking systems are particularly useful in this application, since they have no line-of-sight issues, so that enclosures and screens can be placed around the monitor if required for an experiment.

\section{Using VRVision}

There are two ways to call VRVision. The first is simply to call the main function, without any parameters, by typing "vrvision" or its abbreviated name, "vrvis," within MATLAB:

$>>$ vrvis.

This starts the main VRVision program and displays the current 3-D scene, which is specified in the configuration file "vrvision.ini." However, some parameters can be set before calling VRVision - for example, to specify which scene file to load:

>> vrvis('SetFilename', 'scene.iv').

This loads a file called "scene.iv" from the present working directory. The path to a specific directory can be specified here, if preferred.

VRVision has a collection of functions like the example above. In general, each function consists of a call to VRVision, with the name of the function, followed by an optional list of parameters.

Some functions allow parameters to be set before VRVision runs, whereas others allow results to be retrieved when it has finished running. For example, it is possible to determine whether a key was pressed:

$$
\text { >> vrvis('UseQuitKeys', '12'). }
$$

This will cause VRVision to stop and return to MATLAB when the number key " 1 " or " 2 " is pressed. The time at which the key was pressed will also be recorded and is available to the MATLAB script.

There are 20 functions in total, including the following features: (1) adjust the position of the viewer and scene, relative to the monitor, (2) alter the scale (size) of scene, (3) rotate the scene under script control, (4) detect keypresses, with timing information, (5) allow the user to manipulate the scene with the mouse, (6) record

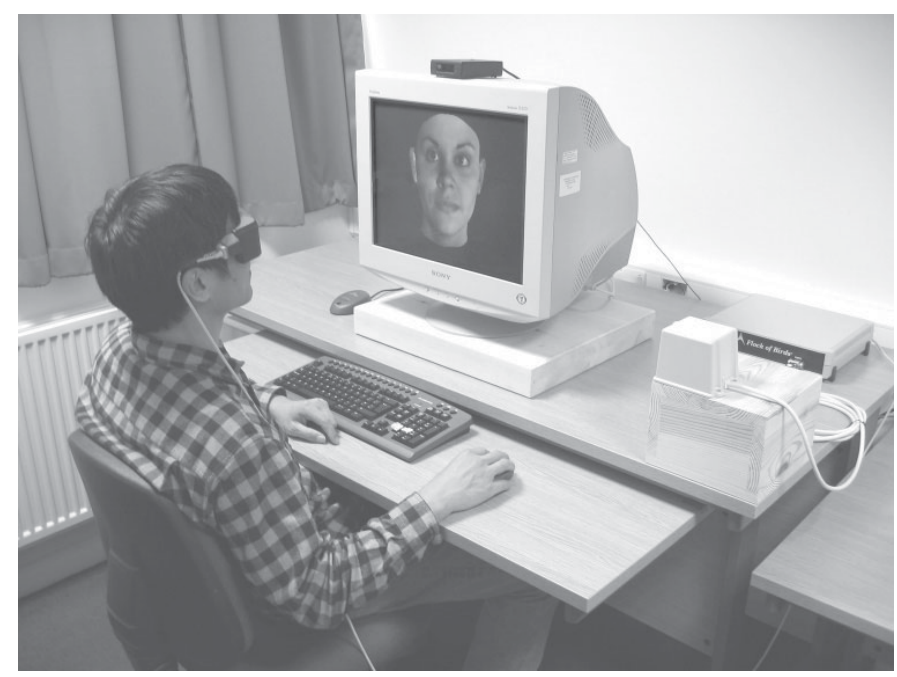

Figure 1. Typical experimental setup. The subject is wearing LCD glasses (CrystalEyes), and the tracking system (Flock of Birds) is mounted on the wooden block at the right side. The infrared emitter for the glasses is placed on top of the monitor. 
mouse movement to a file and replay later, (7) change the background color, and (8) display background images.

These functions are described in more detail in the user manual, which accompanies the program, along with examples of their use.

In addition to the MATLAB script functions that VRVision provides, many different effects can be achieved by modifying the Open Inventor scene files. For example, it is possible to describe animation within an Inventor file, such as an object that follows a predetermined path. The Open Inventor format is mature and well documented - in particular, by the Inventor Mentor (Wernecke, 1994).

\section{Examples of Application}

As an example of a VRVision application, we will consider an experiment in which a number of 3-D shapes need to be displayed under different conditions of rotation.

Conventionally, this might be implemented by developing custom application software to display the 3-D shapes or by preparing the rotated shapes in advance with a modeling package, such as 3D Studio MAX, and then displaying the rendered shapes as a sequence of bitmap images. In this case, it would be necessary to prepare an image for each separate rotation condition, leading to a large set of images.

In contrast, VRVision makes it possible to display the 3 -D shapes directly on the screen. The conditions can be changed under control of a MATLAB script. For example, the MATLAB script below would load a particular shape from file, rotate it $45^{\circ}$ about the $y$-axis (which is vertical), and display it:

$$
\begin{aligned}
& \text { >> vrvis('SetFilename', 'Shape3.iv') } \\
& \text { > > vrvis('SetAngleY', 45) } \\
& \text { > > vrvis. }
\end{aligned}
$$

In practice, the filename and rotation angle could be substituted by variables. Simply by enclosing this program code in a loop, a large number of conditions can be displayed without the need to manually model each condition.

We have already used VRVision successfully in a face recognition study (Liu, Ward, \& Young, in press), where 3-D laser-scanned face models were displayed at different angles with or without stereopsis. An example of a face model rendered at different angles is shown in Figure 2. Each face model used in the study consists of a high-resolution polygon surface mesh with a color photographic texture mapped onto the surface. The face models were converted to the Open Inventor file format in order to be displayed by VRVision within MATLAB.

Let us now consider a slightly more advanced example, which involves using an Open Inventor file. This file format allows 3-D objects to be described in a plain text format. Common geometrical primitives, such as cubes and
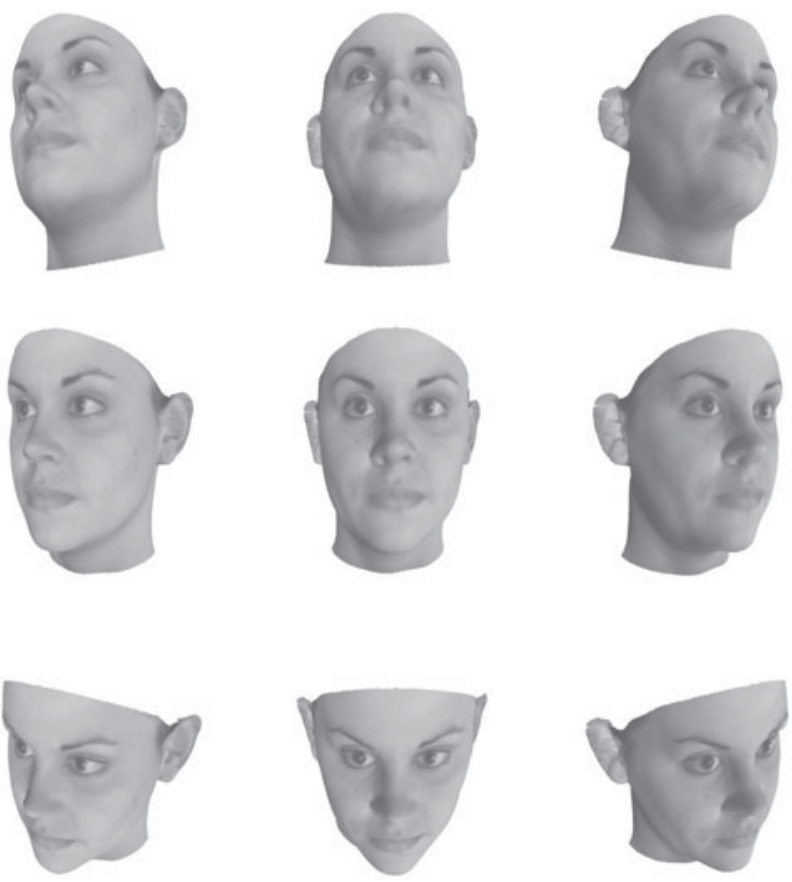

Figure 2. Single laser-scanned face model displayed by VRVision in nine different orientations, by combining rotations on the $x$ - and $y$-axes $\left(-30^{\circ}, 0^{\circ},+30^{\circ}\right)$. The face is displayed using the original photo texture without additional lighting. 
cylinders, are described very simply in the Inventor file; for example, a cylinder could be described as follows:

$$
\begin{aligned}
& \text { Cylinder }\{ \\
& \text { radius } 5 \\
& \text { height } 20
\end{aligned}
$$

The units of measure can be metric or imperial, since the overall scale of the scene can be configured within VRVision. More complex surfaces are described as a list of triangles that are defined by the 3-D coordinates of their vertices or corner points. Some examples are provided in the VRVision documentation.

Therefore, it is possible for a MATLAB script to generate objects directly in this format and output them to a file. These can then be displayed in 3-D by the VRVision program. An example is shown in Figure 3. This means that a MATLAB script can generate an arbitrary number of stimuli (in this case, a 3-D scene) by random variation of parameters. The only tools needed to achieve this are MATLAB and VRVision. The stimuli can be generated from a normal MATLAB program, so that no extensive programming knowledge is needed. This has been possible for some time with 2-D shapes but would previously have been more difficult to achieve with 3-D scenes.

\section{Future Work}

With a suitable graphics card, VRVision is capable of high-quality rendering, including high-resolution textures and antialiasing. However, as with any real-time graphics application, it does not match the quality that can be achieved with rendering packages such as 3D Studio MAX (Discreet/Autodesk Inc.) or Maya (Alias Wavefront) or with global illumination renderers such as RADIANCE (Ward, 1994) and FinalRender (Cebas Computer $\mathrm{GmbH})$.

This limitation may not be relevant in cases in which purely synthetic shapes are displayed but may be of concern when a highly realistic representation of a real-world object is needed. In particular, VRVision currently cannot display cast shadows. The lighting model is computed only at polygon vertices, whereas a rendering package will compute lighting on a per pixel basis.

With highly detailed polygon models-for example, laser-scanned face models (Figure 4) - the difference
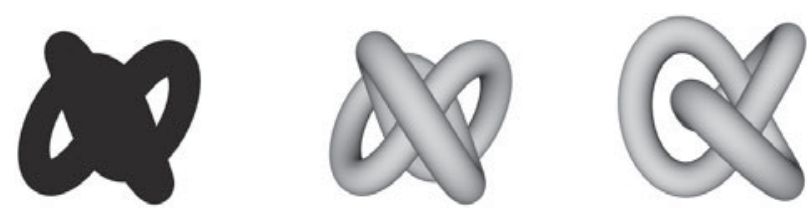

Figure 3. Example of a single synthetic object model, a torus knot, displayed by VRVision as a silhouette, with shading, and after a $45^{\circ}$ rotation. Since objects of this type can be described parametrically, they can be generated by a MATLAB script.
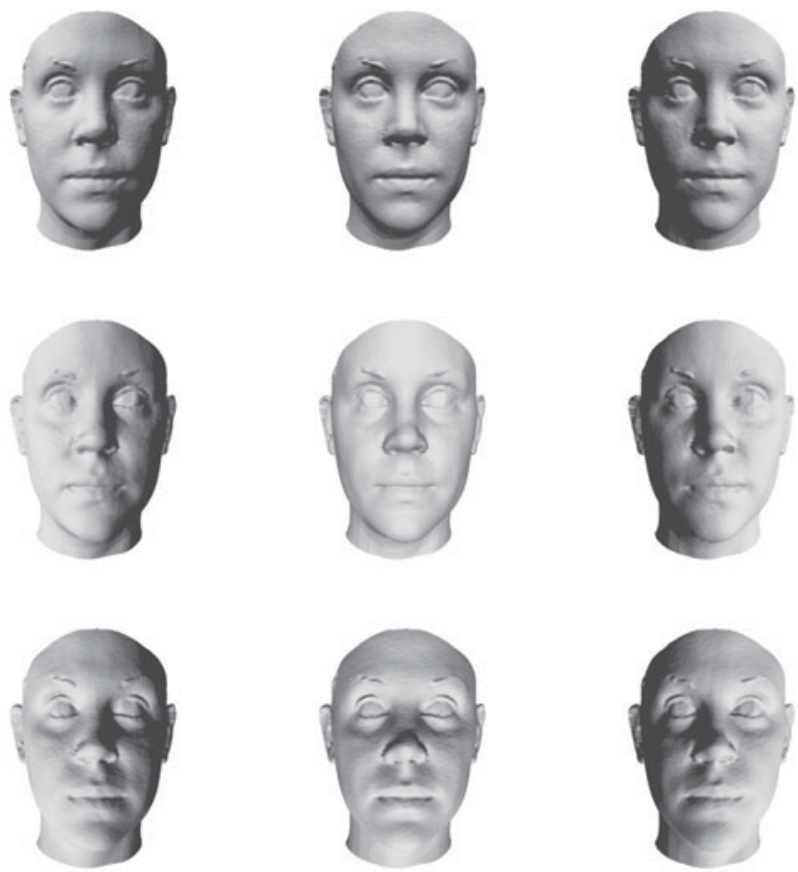

Figure 4. The same face model displayed by VRVision under nine different lighting directions, without photo texturing. The lighting directions are the result of combined rotations $\left(-45^{\circ}\right.$, $0^{\circ},+45^{\circ}$ ) on the $x$ - and $y$-axes.

between per vertex and per pixel lighting models is less significant. However, shadowing can be achieved at present only by using preprepared texture maps that include shadows.

In the future, it will be possible to incorporate shadowing into VRVision and to improve lighting quality. There are several competing initiatives to improve the shading capabilities of real-time graphics cards, such as the HLSL (High Level Shading Language), the OpenGL Shading Language and the language CG (NVidia Corp.). These provide a route for implementing more complex lighting models, shadowing, and numerous other visual effects in the future.

\section{Conclusions}

VRVision provides a new method for displaying 3-D scenes from within MATLAB, which supplements existing tools such as the Psychophysics Toolbox. It allows a wide range of experimental conditions to be realized without the need to prerender and store images or develop custom software.

This software brings capabilities such as stereoscopic display and head tracking, which are normally difficult or expensive to achieve, to a wider community of users.

\section{REFERENCES}

AKKA, R. (1998). Writing stereoscopic software for StereoGraphics systems using Microsoft Windows OpenGL. San Rafael, CA: StereoGraphics Corporation. 
Brainard, D. H. (1997). The Psychophysics Toolbox. Spatial Vision, 10, 433-436.

Liu, C. H., \& Chaudhuri, A. (2003). Face recognition with perspective transformation. Vision Research, 43, 2393-2402.

Liu, C. H., Collin, C. A., \& Chaudhuri, A. (2000). Does face recognition rely on encoding of 3-D surface? Examining the role of shapefrom-shading and shape-from-stereo. Perception, 29, 729-743.

Liu, C. H., WARD, J., \& Young, A. W. (in press). Transfer between 2D and 3D representations of faces. Visual Cognition.

Pelli, D. G. (1997). The VideoToolbox software for visual psychophysics: Transforming numbers into movies. Spatial Vision, 10, 437-442.
WARD, G. J. (1994). The RADIANCE Lighting Simulation and Rendering System. In Computer Graphics, SIGGRAPH'94 Conference Proceedings (pp. 459-472). New York: ACM Press.

Wernecke, J. (1994). The inventor mentor: Programming objectoriented 3-D graphics with Open Inventor, Release 2. Boston: Addison-Wesley.

(Manuscript received May 15, 2004;

revision accepted for publication September 8, 2004.) 